\title{
Decreased Muscle Strength of Knee Flexors is Associated with Impaired Muscle Insulin Sensitivity in Non-Diabetic Middle-Aged Japanese Male Subjects
}

\author{
Yuki Someya $\cdot$ Yoshifumi Tamura $\cdot$ Kageumi Takeno $\cdot$ Saori Kakehi · Takashi Funayama \\ Yasuhiko Furukawa · Hiroaki Eshima - Keisuke Watanabe · Toshiyuki Kurihara · Toshio Yanagiya • \\ Hideyoshi Kaga · Ruriko Suzuki · Daisuke Sugimoto · Satoshi Kadowaki • Ryuzo Kawamori • \\ Hirotaka Watada
}

Received: May 24, 2020 / Published online: August 7, 2020

(C) The Author(s) 2020

\begin{abstract}
Introduction: Reduced muscle strength is a high risk factor for type 2 diabetes mellitus, and this association is especially strong in non-obese male individuals. However, it remains unclear how reduced muscle strength affects susceptibility to diabetes. We have examined whether lower limb muscle strength is associated with insulin resistance in non-obese Japanese male subjects.
\end{abstract}

Digital Features To view digital features for this article go to https://doi.org/10.6084/m9.figshare.12696587.

Y. Someya · Y. Tamura · K. Takeno · S. Kakehi ·

H. Eshima · R. Kawamori · H. Watada

Sportology Center, Juntendo University Graduate

School of Medicine, 2-1-1 Hongo, Bunkyo-ku, Tokyo

113-8421, Japan

Y. Tamura $(\varangle) \cdot$ K. Takeno · T. Funayama ·

Y. Furukawa $\cdot$ H. Kaga $\cdot$ R. Suzuki · D. Sugimoto ·

S. Kadowaki · R. Kawamori · H. Watada

Department of Metabolism and Endocrinology, Juntendo University Graduate School of Medicine, 2-1-1 Hongo, Bunkyo-ku, Tokyo 113-8421, Japan e-mail: ys-tamur@juntendo.ac.jp

Y. Tamura

Faculty of International Liberal Arts, Juntendo

University, 2-1-1 Hongo, Bunkyo-ku,

Tokyo 113-8421, Japan

Y. Someya - K. Watanabe - T. Kurihara - T. Yanagiya Graduate School of Health and Sports Science, Juntendo University, 1-1 Hiraga-gakuendai, Inzai City, Chiba 270-1695, Japan
Methods: Measurements from 64 non-diabetic, non-obese, middle-aged Japanese men were analyzed. Insulin sensitivity in muscle was measured using the hyperinsulinemic-euglycemic clamp. Isometric muscle strength of the knee extensor and flexor muscles was evaluated using a dynameter.

Results: Lower muscle strength of knee flexors, but not knee extensors, was associated with impaired muscle insulin sensitivity (knee flexor muscles: low, medium, and high strength was $6.6 \pm 2.2,7.3 \pm 2.0$, and $8.8 \pm 2.2 \mathrm{mg} / \mathrm{kg}$ per minute, respectively, $p$ for trend $<0.05$; knee extensor muscles: low, medium, and high strength was $7.3 \pm 2.5, \quad 7.5 \pm 2.2$, and $7.8 \pm 2.3 \mathrm{mg} / \mathrm{kg}$ per minute, respectively, $p$ for trend $=0.73$ ). Knee flexor muscle strength was also identified as an independent determinant of insulin sensitivity in the multiple regression analysis $(\beta=0.274, p=0.036)$.

Conclusions: Diminished strength of knee flexor muscles, but not knee extensor muscles, was associated with muscle insulin sensitivity in non-diabetic, non-obese Japanese male subjects.

Keywords: Insulin sensitivity; Muscle strength; Non-obese 


\section{Key Summary Points}

The exact mechanisms of impaired muscle strength in persons with type 2 diabetes are still unclear.

The aim of this study was to examine whether lower limb muscle strength is associated with insulin resistance in nondiabetic men.

The results showed that lower limb flexor muscle strength, but not extensor muscle strength, was associated with insulin sensitivity in muscle.

Muscle strength weakness was also associated with insulin resistance before the onset of type 2 diabetes.

\section{INTRODUCTION}

Reduced muscle strength is a risk factor for type 2 diabetes mellitus (T2DM) [1], and muscle strength is lower in patients with T2DM than in their non-diabetic counterparts [2]. In addition, reduced muscle strength predicts cardiovascular disease and all-cause mortality in communitydwelling populations [3] and patients with T2DM [4]. Thus, enhancing muscle strength may help prevent the onset of T2DM and improve prognosis in pre-diabetic subjects as well as patients with T2DM. While reduced muscle strength in T2DM can be caused both by decreased muscle mass [5] and impaired muscle contractile function [6], the exact mechanism and time course of the latter in patients with T2DM are still unclear. Previous studies have demonstrated that decreased knee extensor muscle strength, adjusted by muscle mass [7] or lean body mass [8], is associated with insulin resistance as determined by the homeostasis model assessment of insulin resistance (HOMAIR) in non-diabetic elderly [7] and healthy younger individuals [8]. These data suggest that insulin resistance that is closely associated with decreased muscle contractile function may be present prior to T2DM onset. However, these previous studies used HOMA-IR as a surrogate marker for whole-body insulin resistance [7] and did not precisely evaluate insulin sensitivity using the hyperinsulinemic euglycemic clamp, the gold standard for assessing tissue-specific insulin sensitivity. In another study, HOMA-IR was found to be only weakly correlated with clamp-determined muscle insulin sensitivity $(r=-0.63)$ and was not correlated with hepatic insulin sensitivity [9]. Thus, it remains unclear whether insulin sensitivity determined by hyperinsulinemic euglycemic clamp is associated with muscle strength in non-diabetic subjects.

In this context, we designed the present study to investigate the association between muscle strength and muscle insulin sensitivity as determined by hyperinsulinemic euglycemic clamp in non-diabetic, middle-aged Japanese men.

\section{METHODS}

\section{Study Subjects}

We enrolled 70 subjects with a body mass index (BMI) of $\geq 23.0$ to $<25.0 \mathrm{~kg} / \mathrm{m}^{2}$ from the baseline dataset of the Sportology Center Core Study, a single-center, prospective, observational study on the mechanisms underlying metabolic abnormalities in non-diabetic, nonobese, middle-aged (aged 30-50 years) Japanese men [9]. We included those subjects with BMI between 23 and $25 \mathrm{~kg} / \mathrm{m}^{2}$ based on the World Health Organization's proposal of BMI $\geq 23 \mathrm{~kg} / \mathrm{m}^{2}$ and $\mathrm{BMI} \geq 25 \mathrm{~kg} / \mathrm{m}^{2}$ being the thresholds of overweight and obesity, respectively, in Asians [10], with Asian males with BMI ranging from 23 to $25 \mathrm{~kg} / \mathrm{m}^{2}$ supposed to be classified as "at risk" [10]. Subjects who were being treated for hypertension, lipid disorders, diabetes, cardiovascular disease, chronic lung disease, cancer, renal failure, serious hepatic dysfunction, hepatitis B, and hepatitis C were excluded from the study. No muscle strength data were available for six of the 70 subjects who participated in the study; consequently, 
data on 64 subjects were included in this analysis. All individuals gave written informed consent to participate in this study, which was approved by the ethics committee of Juntendo University and conducted according to the principles of the Declaration of Helsinki (2008).

\section{Study Design}

The design of the Sportology Center Core Study has been described previously in detail [11]. Briefly, all subjects underwent a baseline evaluation which included screening conducted in examinations carried out in three visits. In the examinations, the subject underwent oral glucose tolerance test, peak oxygen uptake $\left(\mathrm{VO}_{2-}\right.$ peak) test, lower limb muscle strength test, brieftype self-administered diet history questionnaire, and measurement for daily physical activity level by an accelerometer (Lifecorder; Suzuken, Nagoya, Japan). We also evaluated intramyocellular lipid (IMCL) and intrahepatic lipid (IHL) by ${ }^{1} \mathrm{H}$-magnetic resonance spectroscopy (MRS), total body fat content and fatfree mass (FFM) using the bioimpedance method (InBody; BIOSPACE, Tokyo, Japan), and visceral fat area (VFA) and subcutaneous fat area (SFA) by magnetic resonance imaging (MRI). Each subject then underwent two-step euglycemic hyperinsulinemic clamp tests; each step lasted $180 \mathrm{~min}$, with constant insulin infusions of 10 and $20 \mathrm{mU} / \mathrm{m}^{2} / \mathrm{min}$. Glucose disappearance during the second step was used as an index of muscle insulin sensitivity.

\section{Two-Step Hyperinsulinemic Euglycemic Clamp Test}

The subjects were instructed to consume a weight-maintenance standard diet and limit their daily physical activity to their mean daily physical activity level $\pm 10 \%$ during the 3 days immediately before the clamp test. After an overnight fast, a two-step hyperinsulinemic euglycemic glucose clamp was performed with an artificial endocrine pancreas (STG 22; Nikkiso, Shizuoka, Japan) with glucose tracer. Briefly, after securing an intravenous cannula in the forearm, a bolus dose $\left[200 \mathrm{mg} / \mathrm{m}^{2}\right.$ body surface area (BSA)] of $\left[6,6-{ }^{2} \mathrm{H}_{2}\right]$ glucose (Cambridge Isotope Laboratories, Tewksbury, MA, USA) was injected intravenously. Constant infusion was continued at a rate of $2 \mathrm{mg} / \mathrm{m}^{2} \mathrm{BSA}$ per minute for $3 \mathrm{~h}(-180$ to $0 \mathrm{~min}$ ) to measure endogenous glucose production (EGP) at fasting state [12]. This was followed by a primed insulin infusion $\left(40 \mathrm{mU} / \mathrm{m}^{2}\right.$ per minute followed by $20 \mathrm{mU} / \mathrm{m}^{2}$ per minute, each lasting $5 \mathrm{~min}$ ) and continuous insulin infusion at $10 \mathrm{mU} / \mathrm{m}^{2}$ per minute for $3 \mathrm{~h}$ (first step) (0-180 min). In the second step of the clamp, after a priming insulin infusion $\left(80 \mathrm{mU} / \mathrm{m}^{2}\right.$ per minute followed by $40 \mathrm{mU} / \mathrm{m}^{2}$ per minute, each lasting $5 \mathrm{~min}$ ), insulin was infused continuously at $20 \mathrm{mU} / \mathrm{m}^{2}$ per minute for $3 \mathrm{~h}$ (180-360 min). We used a warming blanket for arterialization of the hand vein, and plasma glucose level in arterialized blood was maintained at approximately $95 \mathrm{mg} /$ dl by a variable $20 \%$ glucose infusion containing approximately $2.5 \%\left[6,6-{ }^{2} \mathrm{H}_{2}\right]$ glucose. Blood samples were obtained for biochemical analysis at 10-min intervals during the last $30 \mathrm{~min}$ of the steady-state period of the first and second steps of the clamp. Enrichment of $\left[6,6-{ }^{2} \mathrm{H}_{2}\right]$ glucose in plasma was measured by high-performance liquid chromatography (LTQ-XL-Orbitrap mass spectrometer; Thermo Fisher Scientific, Waltham, MA, USA) as described previously [9].

\section{Calculations}

A steady-state equation was used to calculate the rates of EGP and glucose disappearance (Rd) at each step [9]. EGP and Rd were normalized by BSA and FFM, respectively [9]. We calculated percentage reduction of EGP at the first step and used the result as an index of hepatic insulin sensitivity [13]. Rd at the second step was used as an index of muscle insulin sensitivity [14].

\section{${ }^{1} \mathrm{H}$-MRS and MRI}

The IMCL values of the right tibialis anterior and soleus muscles and the IHL of segment 6 in the liver were measured by ${ }^{1} \mathrm{H}$-MRS (VISART EX V4.40; Toshiba, Tokyo, Japan) $[15,16]$. After the measurements, IMCL was quantified by methylene signal intensity (S-fat) using the 
creatine signal (Cre) as the reference, and calculated as the ratio S-fat/Cre. IHL was quantified by $\mathrm{S}$-fat using $\mathrm{H}_{2} \mathrm{O}$ as the internal reference, and calculated as the percentage of $\mathrm{H}_{2} \mathrm{O}+\mathrm{S}$-fat [Sfat $\times 100 /\left(\mathrm{H}_{2} \mathrm{O}+\mathrm{S}\right.$-fat $\left.)\right][15,16]$. VFA and SFA were measured with MRI as described previously [16]. Briefly, T1-weighted trans-axial scans were obtained, and VFA and SFA at the fourth and fifth lumbar interspaces were measured as described previously using specific software (AZE Virtual Place, Tokyo, Japan) [16].

\section{Knee Extensor/Flexor Muscle Strength Test}

Knee extensor/flexor muscle strength was measured using the Cybex770 system (Cybex Division of Lumex, Ronkonkoma, NY, USA). The isometric peak torques of knee extensors/flexors were measured at an angular velocity of $60^{\circ}$ for $30 \mathrm{~s}$. During the test, participants were encouraged to perform maximal muscle contractions. The isometric peak torques $(\mathrm{Nm})$ of knee extensors/flexors were adjusted by body weight (kg).

\section{Statistical Analysis}

Study subjects were categorized into tertiles of low, medium, and high, respectively, according to knee extensor/flexor muscle strength. Data are presented as mean \pm standard deviation. Data of the three groups were compared by oneway analysis of variance. The dose-response relationship in the three groups was analyzed using the Jonckheere Terpstra test. Multiple linear regression was used to analyze the relationship between muscle insulin sensitivity and muscle strength and other risk factors. This study included adiponectin, VFA, and $\mathrm{VO}_{2 \text { peak }}$ as covariates, all of which were correlated with muscle insulin sensitivity in our previous study [9]. All statistical tests were two-sided with a 5\% level of significance.

\section{RESULTS}

The physical characteristics of the subjects categorized by muscle strength tertile are summarized in Tables 1 and 2. The muscle strength of knee extensors was approximately threefold greater than that of knee flexors, and both strengths were positively associated with each other. Both strengths were negatively associated with percentage body fat, VFA, and SFA, and were positively associated with FFM ( $p$ for trend $<0.05$ ). Although both muscle strengths were associated with several indices representing adiposity, only knee flexor muscle strength was negatively associated with elevated free fatty acids, fasting plasma insulin and HOMA-IR, and reduced adiponectin. Further, knee flexor muscle strength was positively associated with insulin sensitivity in muscle. In contrast, knee extensor muscle strength was not associated with insulin sensitivity in muscle. These same relations between muscle strength and insulin sensitivity were observed when muscle strength was adjusted by FFM instead of body weight. In addition, we investigated the relationship between muscle strength and insulin sensitivity using multiple linear regression analysis. Due to the small number of study subjects, we included a limited number of covariates in the multiple regression analysis: age, muscle strength, visceral fat area, adiponectin, and $V \mathrm{O}_{2 \text { peak }}$. Knee flexor muscle strength $(\beta=0.274, p=0.036)$ and adiponectin ( $\beta=0.239, p=0.042)$ were identified as independent determinants of insulin sensitivity (Table 3, Model 1). When we included free fatty acids instead of adiponectin in the model, only knee flexor muscle strength was significant $(\beta=0.277, p=0.041)$ (Table 3, Model 2).

\section{DISCUSSION}

The results of the present study show that in this population of non-obese, non-diabetic Japanese male subjects, reduced knee flexor muscle strength was associated with impaired insulin sensitivity in muscle. Interestingly, knee extensor muscle strength was not associated with insulin sensitivity in muscle in this population.

Reduced muscle strength of the knee flexors, but not the knee extensors, was associated with muscle insulin resistance as determined by 


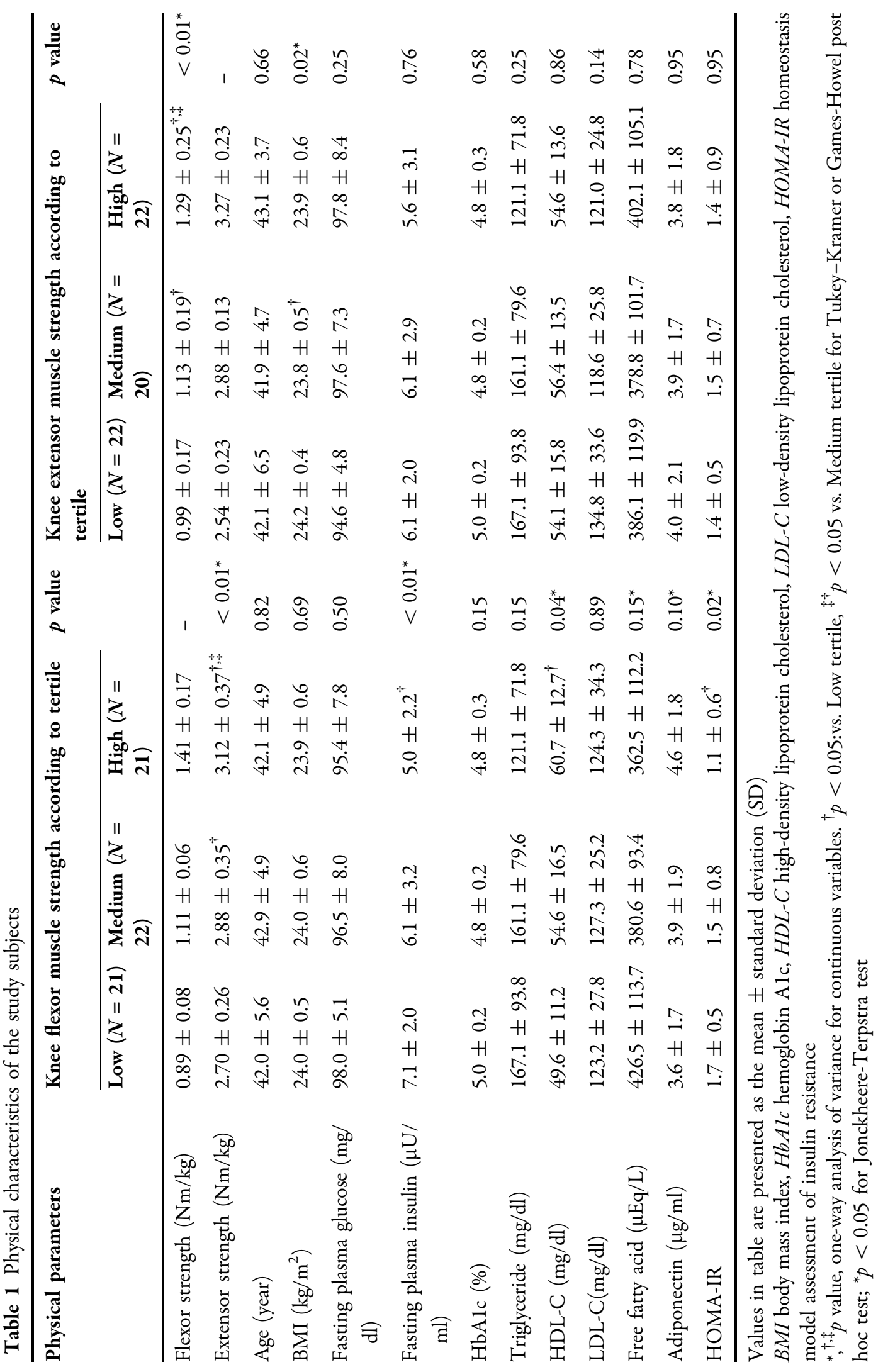




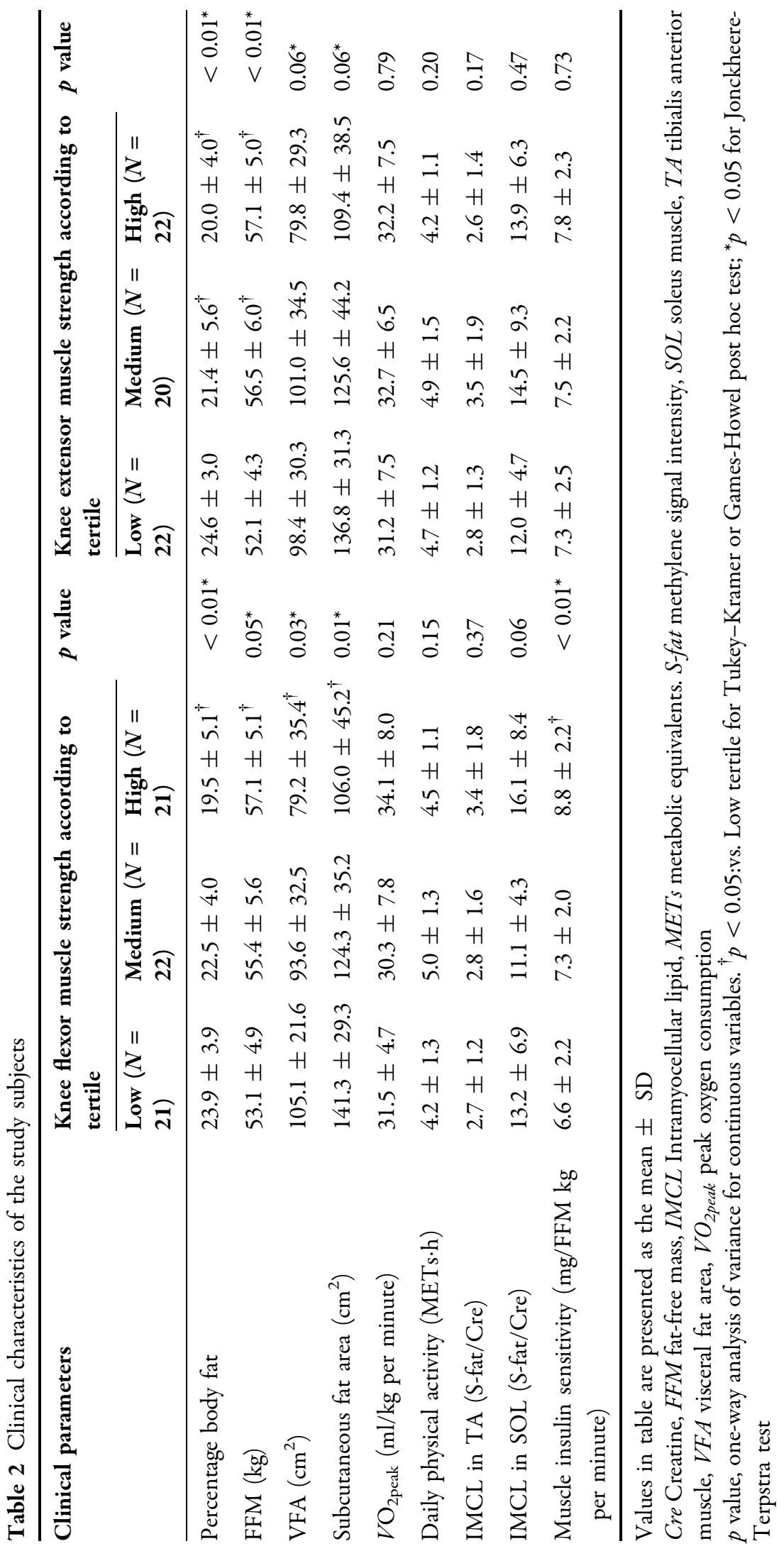


Table 3 Multiple linear regression for relationship between muscle strength and insulin sensitivity

\begin{tabular}{|c|c|c|c|c|}
\hline \multirow[t]{2}{*}{ Muscle strength and model covariates } & \multicolumn{2}{|c|}{ Knee flexor muscle strength } & \multicolumn{2}{|c|}{ Knee extensor muscle strength } \\
\hline & $\beta$ & $p$ value & $\bar{\beta}$ & $p$ value \\
\hline \multicolumn{5}{|l|}{ Model 1} \\
\hline Flexor strength $(\mathrm{Nm} / \mathrm{kg})$ & 0.274 & 0.036 & - & - \\
\hline Extensor strength $(\mathrm{Nm} / \mathrm{kg})$ & - & - & 0.060 & 0.477 \\
\hline Age & -0.002 & 0.990 & 0.071 & 0.586 \\
\hline Adiponectin $(\mu \mathrm{g} / \mathrm{ml})$ & 0.239 & 0.042 & 0.286 & 0.021 \\
\hline VFA $\left(\mathrm{cm}^{2}\right)$ & -0.164 & 0.223 & -0.244 & 0.081 \\
\hline$V \mathrm{O}_{2 \text { peak }}(\mathrm{ml} / \mathrm{kg}$ per minute $)$ & 0.176 & 0.182 & 0.223 & 0.104 \\
\hline \multicolumn{5}{|l|}{ Model 2} \\
\hline Flexor strength $(\mathrm{Nm} / \mathrm{kg})$ & 0.277 & 0.041 & - & - \\
\hline Extensor strength $(\mathrm{Nm} / \mathrm{kg})$ & - & - & 0.029 & 0.827 \\
\hline Age & -0.008 & 0.994 & 0.076 & 0.588 \\
\hline Free fatty acids $(\mu \mathrm{Eq} / \mathrm{l})$ & -0.072 & 0.562 & -0.097 & 0.458 \\
\hline $\operatorname{VFA}\left(\mathrm{cm}^{2}\right)$ & -0.230 & 0.109 & -0.333 & 0.024 \\
\hline$V \mathrm{O}_{2 \text { peak }}(\mathrm{ml} / \mathrm{kg}$ per minute $)$ & 0.175 & 0.214 & 0.225 & 0.128 \\
\hline
\end{tabular}

$p$ value: multiple linear regression analysis adjusted for all factors in the table

hyperinsulinemic euglycemic clamp. In contrast, a study using HOMA-IR showed that reduced knee extensor muscle strength was associated with insulin resistance in the elderly [7] and that muscle strength of both the knee flexors and extensors was associated with insulin resistance in healthy young subjects [8]. The Rd used as an index of muscle insulin sensitivity in the present study was only weakly correlated with HOMA-IR $(r=-0.63)$ [11]. In addition, in the present study we analyzed only non-obese Japanese male subjects, which differs from the study populations in previous studies in terms of age and BMI $[7,8]$. These variations in subjects and methods used to evaluate insulin sensitivity may explain the differences in study results.

Similar to previous studies in non-diabetic elderly and young subjects $[7,8]$, we observed a link between decreased muscle strength and muscle insulin resistance. Results from an animal model also showed that muscle contractile function was reduced in parallel with impaired insulin sensitivity during administration of a high-fat diet before diabetes onset [17]. Regarding the causal relation between these factors, a meta-analysis showed that resistance training, which increases muscle strength, improved markers of insulin resistance in subjects with metabolic syndrome [18]. On the other hand, Marsh et al. investigated the effect of an insulin sensitizer, pioglitazone, on knee extensor muscle strength and leg muscle power during voluntary weight loss in non-diabetic older adults [19] and showed that in women, pioglitazone potentiated the effect of resistance training on muscle power but not muscle strength, and did not alter lean body mass. These data suggest that improving insulin resistance might enhance muscle function independent of muscle mass change, an approach that may be applicable to diabetic patients with reduced muscle strength. In fact, muscle strength has been reported to be reduced in diabetic patients [20]. 
It is still unclear why the strength of knee flexors, but not extensors, was associated with insulin sensitivity in this study. It has been shown that type I muscle fibers have a higher glucose-handling capacity than type II muscle fibers [21]. Interestingly, results from an animal model recently showed that muscle strength was reduced in parallel with insulin resistance during a high-fat diet, but that this occurred only in the extensor digitorum longus muscle, which contains predominantly type II fibers, and not in the soleus muscle, which comprises mainly type I fibers [17]. Knee flexor muscles have been reported to have a relatively high proportion of type II fibers [22], and thus the strength of knee flexors is more likely to be associated with insulin sensitivity than that of extensors. In addition, knee extensors are generally used more during physical activity than knee flexors, and thus the association between insulin sensitivity and knee extensor muscle strength might be diminished by major covariates, such as physical activity. Taken together, decreased knee flexion muscles strength might be a better marker for impaired muscle insulin sensitivity than knee extensor muscle strength.

The present study has several limitations. First, the analysis was conducted on only Japanese male subjects. Since glucose and lipid metabolism and fat distribution vary by sex and ethnicity, our results may not be applicable to females and other ethnic groups. Another possible limitation is the relatively small number of subjects. However, two-step hyperinsulinemic euglycemic clamp is very time consuming (approx. $10 \mathrm{~h}$ ), and therefore the inclusion of more than 60 non-diabetic subjects may represent a strength rather than a limitation. Finally, intervention studies are clearly required to confirm the causality of the parameters identified in the present study.

\section{CONCLUSION}

Knee flexor muscle strength was associated with muscle insulin sensitivity in non-diabetic, middle-aged Japanese male subjects. Further studies are needed to generalize these findings.

\section{ACKNOWLEDGEMENTS}

We thank Mutsuko Yoshikawa, Miyuki Iwagami, Naoko Daimaru, and Emi Miyazawa for their excellent technical assistance. We also thank Hikari Taka and Tsutomu Fujimura (Juntendo University) for performing the LC-MS analysis.

Funding. This study was funded by a High Technology Research Center Grant, Strategic Research Foundation at Private Universities and KAKENHI (23680069, 26282197, 17K19929) from the Ministry of Education, Culture, Sports, Science and Technology of Japan; Japan Diabetes Foundation; Suzuken Memorial Foundation; Mitsukoshi Welfare Foundation; and Diabetes Masters Conference. The Rapid Service Fee was funded by the authors.

Authorship. All named authors meet the International Committee of Medical Journal Editors (ICMJE) criteria for authorship for this article, take responsibility for the integrity of the work as a whole, and have given their approval for this version to be published.

Authorship Contributions. YS, YT, and KT performed the research and contributed to the study design, data collection, interpretation of results, and writing and editing of the manuscript. YS, KT, SK, TF, YF, HE, KW, TK, TY, HK, DS, SK, and RS participated in data collection and analysis, and contributed to the discussion. RK contributed to the discussion. HW contributed to the study design and reviewed and edited the manuscript.

Disclosures. Yuki Someya, Yoshifumi Tamura, Kageumi Takeno, Saori Kakehi, Takashi Funayama, Yasuhiko Furukawa, Hiroaki Eshima, Keisuke Watanabe, Toshiyuki Kurihara, Toshio Yanagiya, Hideyoshi Kaga, Ruriko Suzuki, Daisuke Sugimoto, Satoshi Kadowaki, Ryuzo Kawamori and Hirotaka Watada have nothing to disclose.

Compliance with Ethics Guidelines. All individuals gave written informed consent to participate in this study, which was approved by 
the ethics committee of Juntendo University and conducted according to the principles of the Declaration of Helsinki (2008).

Data Availability. All data generated or analyzed during this study are included in this published article.

Open Access. This article is licensed under a Creative Commons Attribution-NonCommercial 4.0 International License, which permits any non-commercial use, sharing, adaptation, distribution and reproduction in any medium or format, as long as you give appropriate credit to the original author(s) and the source, provide a link to the Creative Commons licence, and indicate if changes were made. The images or other third party material in this article are included in the article's Creative Commons licence, unless indicated otherwise in a credit line to the material. If material is not included in the article's Creative Commons licence and your intended use is not permitted by statutory regulation or exceeds the permitted use, you will need to obtain permission directly from the copyright holder. To view a copy of this licence, visit http://creativecommons.org/licenses/by$\mathrm{nc} / 4.0 /$.

\section{REFERENCES}

1. Tarp J, Stole AP, Blond K, Grontved A. Cardiorespiratory fitness, muscular strength and risk of type 2 diabetes: a systematic review and meta-analysis. Diabetologia. 2019;62(7):1129-42.

2. Park SW, Goodpaster BH, Lee JS, et al. Excessive loss of skeletal muscle mass in older adults with type 2 diabetes. Diabetes Care. 2009;32(11):1993-7.

3. Wu Y, Wang W, Liu T, Zhang D. Association of grip strength with risk of all-cause mortality, cardiovascular diseases, and cancer in community-dwelling populations: a meta-analysis of prospective cohort studies. J Am Med Dir Assoc. 2017;18(6):551e17e35.

4. Celis-Morales CA, Petermann F, Hui L, et al. Associations between diabetes and both cardiovascular disease and all-cause mortality are modified by grip strength: evidence From UK Biobank, a prospective population-based cohort study. Diabetes Care. 2017;40(12):1710-8.

5. Park SW, Goodpaster BH, Strotmeyer ES, et al. Accelerated loss of skeletal muscle strength in older adults with type 2 diabetes: the health, aging, and body composition study. Diabetes Care. 2007;30(6): 1507-12.

6. Park SW, Goodpaster BH, Strotmeyer ES, et al. Decreased muscle strength and quality in older adults with type 2 diabetes: the health, aging, and body composition study. Diabetes. 2006;55(6): 1813-8.

7. Barzilay JI, Cotsonis GA, Walston J, et al. Insulin resistance is associated with decreased quadriceps muscle strength in nondiabetic adults aged $\geq 70$ years. Diabetes Care. 2009;32(4):736-8.

8. Gysel T, Calders P, Cambier D, et al. Association between insulin resistance, lean mass and muscle torque/force in proximal versus distal body parts in healthy young men. J Musculoskelet Neuronal Interact. 2014;14(1):41-9.

9. Takeno K, Tamura Y, Kawaguchi M, et al. Relation between insulin sensitivity and metabolic abnormalities in japanese men with BMI of $23-25 \mathrm{~kg} / \mathrm{m}^{2}$. J Clin Endocrinol Metab. 2016;101(10):3676-84.

10. World Health Organization. The Asia-Pacific perspective: redefining obesity and its treatment: Sydney: Health Communications Australia; 2000.

11. Takeno K, Tamura Y, Kawaguchi M, et al. Relation between insulin sensitivity and metabolic abnormalities in Japanese men with BMI of $23-25 \mathrm{~kg} / \mathrm{m}^{2}$. J Clin Endocrinol Metab. 2016;101(10):3676-84.

12. Kelley DE, McKolanis TM, Hegazi RA, Kuller LH, Kalhan SC. Fatty liver in type 2 diabetes mellitus: relation to regional adiposity, fatty acids, and insulin resistance. Am J Physiol Endocrinol Metab. 2003;285(4):E906-E916916.

13. Kotronen A, Juurinen L, Tiikkainen M, Vehkavaara $\mathrm{S}$, Yki-Jarvinen $\mathrm{H}$. Increased liver fat, impaired insulin clearance, and hepatic and adipose tissue insulin resistance in type 2 diabetes. Gastroenterology. 2008;135(1):122-30.

14. Abdul-Ghani M, DeFronzo RA. Fasting hyperglycemia impairs glucose-but not insulin-mediated suppression of glucagon secretion. J Clin Endocrinol Metab. 2007;92(5):1778-844.

15. Tamura Y, Tanaka Y, Sato F, et al. Effects of diet and exercise on muscle and liver intracellular lipid contents and insulin sensitivity in type 2 diabetic patients. J Clin Endocrinol Metab. 2005;90(6): 3191-6. 
16. Sato F, Tamura Y, Watada H, et al. Effects of dietinduced moderate weight reduction on intrahepatic and intramyocellular triglycerides and glucose metabolism in obese subjects. J Clin Endocrinol Metab. 2007;92(8):3326-9.

17. Eshima H, Tamura $\mathrm{Y}$, Kakehi S, et al. Long-term, but not short-term high-fat diet induces fiber composition changes and impaired contractile force in mouse fast-twitch skeletal muscle. Physiol Rep. 2017;5(7):e13250.

18. Strasser B, Siebert U, Schobersberger W. Resistance training in the treatment of the metabolic syndrome: a systematic review and meta-analysis of the effect of resistance training on metabolic clustering in patients with abnormal glucose metabolism. Sports Med. 2010;40(5):397-415.
19. Marsh AP, Shea MK, Vance Locke RM, et al. Resistance training and pioglitazone lead to improvements in muscle power during voluntary weight loss in older adults. J Gerontol A Biol Sci Med Sci. 2013;68(7):828-36.

20. Andersen H, Nielsen S, Mogensen CE, Jakobsen J. Muscle strength in type 2 diabetes. Diabetes. 2004;53(6):1543-8.

21. Albers PH, Pedersen AJ, Birk JB, et al. Human muscle fiber type-specific insulin signaling: impact of obesity and type 2 diabetes. Diabetes. 2015;64(2): 485-97.

22. Garrett WE Jr, Califf JC, Bassett FH 3rd. Histochemical correlates of hamstring injuries. Am J Sports Med. 1984;12(2):98-103. 